\title{
PERSPECTIVA DE ATENCIÓN PSICOTERAPÉUTICA A PERSONAS EN SITUACIÓN DE REFUGIO
}

\section{Perspective of psychotherapeutic care for people in a refugee situation}

Antonia Lara*

\begin{abstract}
Resumen. El presente artículo tiene como objetivo dar cuenta de una perspectiva de abordaje en la atención psicoterapéutica a refugiados latinoamericanos, construida mediante la sistematización de la experiencia clínica del área de migración, interculturalidad y refugio (AMIR), desde un Centro Universitario. Así, se plantean los ejes centrales de la perspectiva propuesta y se discuten con las tradiciones psicológicas que tienden a la psicopatologización de la experiencia migratoria. Para luego profundizar, a la luz de un caso clínico, acerca de las problemáticas en la instalación de la transferencia en aquellas personas en situación de refugio.
\end{abstract}

Palabras claves: atención psicoterapéutica; refugiados; Chile.

\begin{abstract}
The present article aims to give an account of a perspective of approach in psychotherapeutic care to Latin American refugees, build through the systematization of clinical experience in the area of migration, interculturality and refuge (AMIR), from a university Center. Thus, the central axes of the perspective are presented and discussed with the psychological traditions that tend to psychopathologize the migratory experience. To then deepen, in light of a clinical case, in the problems in the installation of the transference with people in a refugee situation.
\end{abstract}

Keywords: psychotherapeutic care; refugee; Chile.

\section{Introducción}

En las últimas décadas, Chile se ha vuelto un activo receptor de inmigrantes, pasando de tener $1,27 \%$ de su población de origen extranjero (INE, 2002), a 4,35\%, según el Censo del año 2017. A principio de los años 2000, las personas extranjeras provenían principalmente de países como Perú, Argentina y Bolivia. Más tarde, ésta se ha compuesto también de personas provenientes de países como Colombia, Venezuela y Haití.

\footnotetext{
Investigadora y académica del Centro de investigación en Ciencias Sociales y Juventud, de la Universidad Católica Silva Henríquez. Santiago, Chile. E-mail: antonialew@gmail.com. Orcid: 0000-0002-3530-4763.
} 
Estudios han determinado que, "el efecto del migrante sano (población migrante en promedio con menor auto-reporte de problemas de salud que la población local chilena) desaparece en migrantes de nivel socioeconómico bajo, que llevan más de 20 años en Chile" (Cabieses, Bernales, Mclntyre, 2017, p. 19). Si bien lo anterior, no implica un aumento sustantivo en las demandas espontáneas de atención, se ha ido generando paulatinamente una demanda de atención en salud mental, derivada de instituciones que trabajan con inmigrantes en Santiago (Becerra, Altimir, 2013).

En este contexto, en el siguiente artículo se propone, desde la migración sur-sur, profundizar en la reflexión sobre la atención psicoterapéutica a personas en situación de refugio a través de un caso clínico atendido en el Área de migración, interculturalidad y refugio (AMIR), perteneciente a la Universidad Católica Silva Henríquez (UCSH).

El texto comienza contextualizando el escenario de la inmigración a Chile, la cual ha ido en aumento en cantidad, diversidad de países de proveniencia y complejización de los procesos. Así como del Centro universitario, en la cual se realizan los procesos de psicoterapia.

En la segunda parte, se exponen los ejes centrales de la perspectiva de trabajo clínico con inmigrantes y refugiados que realiza AMIR, la cual fue elaborada a partir de la experiencia acumulada en siete años de trabajo clínico del equipo y sistematizada durante el año 2017, por el mismo. Dicha sistematización se llevó a cabo a lo largo de seis meses, a través de reuniones periódicas de reflexión entre los psicólogo/as del área y la facilitadora externa de la sistematización. La reflexión se organizó en torno a las siguientes preguntas: ¿cuáles son las especificidades de la escucha clínica con personas inmigrantes en situación de refugio? y ¿cómo situar la cultura, tanto del paciente como del terapeuta, en la relación clínica? En el presente artículo, exponemos el campo de debates sobre el papel de la cultura en las problemáticas de salud mental que atraviesan los migrantes y la posición crítica que sostenemos respecto a aquellas tradiciones que tienden a la psicopatologización de la experiencia de migración.

A partir de la sistematización realizada se identificó una serie de nudos críticos en la atención psicoterapéutica a inmigrantes y refugiados, que fue plasmada en un artículo de autoría colectiva (Lara et alii, 2017). De aquel artículo retomamos aquí, en la tercera parte, aquel nudo crítico sobre las dificultades en la instalación de la transferencia ${ }^{1}$ cuando se trata de la atención

\footnotetext{
1 La transferencia es un concepto utilizado por Freud $(1895,1901,1912)$ y designa la relación analista y analizado, como dispositivo que permite el despliegue de los conflictos del sujeto y su interpretación. Laplanche y Pontalis (2004, p. 439) lo definen como “(...) el proceso en virtud del cual los deseos inconscientes se actualizan sobre ciertos objetos, dentro de un determinado tipo de relación establecida con ellos y, de un modo especial, dentro de la relación analítica. Se trata
} 
de personas en situación de refugio para, a la luz de un caso clínico, mostrar sus implicancias.

\section{Contexto Nacional e Institucional}

Chile ha sido históricamente un país expulsor de personas, constituyendo los principales destinos de los chilenos en el extranjero, Argentina, Estados Unidos y Suecia (Stefoni, 2011). Sin embargo, con el retorno a la democracia y la estabilidad económica del país, hacia finales de la década de los años '90 comienza a aumentar el flujo de personas provenientes de países latinoamericanos hacia Chile.

El Censo del año 2002 mostró que los nacidos en el extranjero que declararon residir habitualmente en Chile, era el 1,27\%, y que provenían en su mayoría de países limítrofes como Perú en primer lugar, y luego Argentina y Bolivia; entre los cuales, siguiendo la tendencia de la región latinoamericana, un poco más del 50\% eran mujeres (Martínez, 2003). Posteriormente, desde el año 2010, comenzó a incrementar el flujo de personas provenientes de Colombia, Venezuela y Haití. Es así como en el Censo del año 2017 (INE, 2018) se contabilizó que el porcentaje de inmigrantes subió a 4,35\% (contando 746.465 personas) nacidas principalmente en Perú $(25,2 \%)$, Colombia $(14,1 \%)$, Venezuela $(11,1 \%)$, Bolivia $(9,9 \%)$, Argentina $(8,9 \%)$, Haití $(8,4 \%)$ y Ecuador $(3,7 \%)$. En la mayoría de ellos la proporción de hombres y mujeres es similar, con predominio de las mujeres. Así Chile se ha ido configurando como un polo de atracción de inmigrantes provenientes no sólo de países limítrofes, sino también de Centro América, lo cual da cuenta de la diversidad y complejidad en que se han ido desarrollando los procesos migratorios.

Tanto en el Censo del año 2002 como en el del año 2017, se constata que la mayor concentración de inmigrantes se encuentra en la ciudad de Santiago, capital del país, y en particular, en la comuna de Santiago Centro. Esta es la zona administrativa de la ciudad, la cual se caracteriza por construcciones antiguas que son subdivididas y ocupadas por familias de inmigrantes. En esta comuna se encuentra también el Centro de Estudios y Atención a la Comunidad (CEAC) de la Universidad Católica Silva Henríquez (UCSH). CEAC es un centro interdisciplinario de atención psicológica, social y psicopedagógica, en el cual se aloja el área de atención a inmigrantes y refugiados (AMIR). Este es un espacio de intervención y reflexión en torno a las problemáticas subjetivas y de salud mental que presentan las personas en situación de movilidad. Las líneas

de una repetición de prototipos infantiles, vivida con un marcado sentimiento de actualidad. Casi siempre, lo que los psicoanalistas denominan transferencia, sin otro calificativo, es la transferencia en la cura. La transferencia se reconoce clásicamente como el terreno en el que se desarrolla la problemática de una cura psicoanalítica, caracterizándose ésta por la instauración, modalidades, interpretación y resolución de la transferencia. 
de intervención del Área son las de atenciones en psicoterapia (individual, parejas, familias), la realización de talleres grupales con mujeres inmigrantes latinoamericanas orientados a la elaboración de las ilusiones/desilusiones asociadas a sus proyectos migratorios y a la formación de psicólogos en práctica.

Las personas atendidas en AMIR, en su mayoría provienen de instituciones derivantes con las que CEAC mantiene redes y convenios, tales como: Fundación de Ayuda Social de las Iglesias Cristinas (FASIC), Centro Integrado de Atención al Migrante de Santiago (CIAMI), Funda Crisis, Fundación Don Bosco, Consulado del Perú y Colegios de la zona Centro de la ciudad (Santiago-Chile). Quienes llegan a consultar al Centro son adultos, jóvenes, niños y niñas con diversos motivos de emigración, tales como reunificación familiar, económico-laboral, huida de situaciones de violencia intrafamiliar y social, así como también seguir estudios universitarios. Entre quienes consultan en este centro, la mayoría son mujeres adultas, de entre 21 y 50 años, que provienen principalmente de países como Perú, Colombia y en el último tiempo Venezuela.

\section{Perspectiva de trabajo clínico con inmigrantes y refugiados}

Desde su formación en el año 2010, el equipo de atención a refugiados e inmigrantes latinoamericanos en Santiago, ha ido construyendo una perspectiva de abordaje psicoterapéutico del malestar y sufrimiento psíquico, de personas procedentes de diversos países latinoamericanos que llegan a vivir a Santiago de Chile. Esta perspectiva de trabajo clínico, se sostiene en la concepción de que los malestares, sufrimientos y sintomatología de las personas que migran, no son una consecuencia directa de la experiencia migratoria ni se reducen a una etiología cultural, tal como se suele asumir en los abordajes psicoterapéuticos con personas en situación de movilidad.

Como hemos señalado en un texto anterior (Lara et alii, 2017) las producciones sintomáticas que se puedan desplegar en situación de movilidad, como elaboraciones psíquicas posibles, están vinculadas a las condiciones sociales, económicas y políticas tanto de los contextos de salida como en los de arribo; es decir, entramadas con las trayectorias biográficas y los ideales culturales que componen una subjetividad. De tal manera, las sintomatologías que aquejan a los inmigrantes, son el resultado de una configuración compleja de dimensiones heterogéneas, en las que toman mayor preponderancia unas u otras según las condiciones en que se despliega el trayecto migratorio. Es decir, no da lo mismo si las condiciones de movilidad son las de un estudiante becado, de una trabajadora de baja calificación, de un empleado de una empresa transnacional, etc. Tampoco es igual, si el cambio de país ocurre en el marco de un acuerdo bilateral o multilateral que facilita los trámites migratorios y los procesos de instalación en el nuevo país. Por ende, los síntomas y malestares están vinculados a aquellas condiciones sociales, económicas y políticas en que 
se despliega el proceso migratorio, y entramadas a una biografía y subjetividad singular.

De esta manera, desde las concepciones psicoanalíticas de la relación sujeto cultura, nos alejamos de las tradiciones que consideran la experiencia migratoria como causa de trauma psíquico y psicopatología (Nathan, 1999; Atchotegui, 2009; Grinberg, Grinberg, 1996). En la tradición etnopsiquiátrica, se plantea que la salida del sujeto de la cultura de origen, la cual sería fundadora y homeostática del aparato psíquico, tiene un efecto catastrófico para el sujeto (Nathan, 1999). A partir de dicha premisa, desde la perspectiva transcultural, autores como Atchotegui (2009) han llegado a formular una nueva categoría diagnóstica para los migrantes, el Síndrome de Ulises. En esta categoría se describen los síntomas y cuadros clínicos que aquejarían a los migrantes, contribuyendo a la tendencia a psicopatologizar la migración. Como critica Venturini (2006), el problema de dichas concepciones es que suponen la inscripción del sujeto a la cultura como total, como esfera cerrada y originaria, que configura una identidad acabada.

Si bien otras tradiciones en psicología no le atribuyen causalidad psicopatológica a la experiencia migratoria, la conciben en función de dicotomías fijas y excluyentes. De tal manera, tanto Falicov (2015), de la corriente en psicología familiar sistémica, como los psicoanalistas Grinberg \& Grinberg (1996), consideran que la experiencia de migración se despliega exclusivamente en la dimensión de la pérdida, duelo y crisis, donde los conflictos se juegan entre la exclusión/inclusión, pertenencia/extranjeridad, etc., sin abrir espacio a posicionamientos del sujeto migrante que no se despliegan en dichas dicotomías excluyentes.

De manera que si bien las subjetividades, sus malestares y síntomas están inscritos en una cultura, no tienen significación única ni causalidad cultural absoluta. Como ha identificado Brigidi (2009) es una tendencia en los contextos de atención en salud mental, sobresignificar culturalmente los padecimientos de los inmigrantes, Ilegando a dar una atribución nacional a la etiología de los síntomas.

Del mismo modo, hemos planteado que no todos los padecimientos, síntomas y malestares de los sujetos migrantes se explican por la experiencia migratoria, en tanto salida de la cultura de procedencia, sino que se configuran como un entramado complejo donde se ponen en juego dimensiones sociales, económicas, políticas, biográficas y culturales en conflicto; y que en la atención clínica se despliegan de manera singular en cada caso.

Ahora bien, plantear una posición crítica respecto a las tradiciones que tienden a la psicopatologización de la migración, no implica dejar de considerar que en este trayecto pueden surgir síntomas, angustias e inhibiciones, como 
elaboraciones psíquicas de un trayecto en el cual, muchas veces, se reeditan conflictos previos. Sin embargo, lo que planteamos es que aun cuando aquello puede surgir, no necesariamente se va a configurar como cuadro psicopatológico. De tal modo, concebimos que el impacto de la experiencia migratoria en sus protagonistas no es sólo de orden psicopatológico, sino que puede abrir a procesos de subjetivación y reconfiguración identitaria.

El trabajo del sociólogo Sayad (2010) da luces al respecto, al señalar que en el desplazamiento migratorio se le revela al migrante el "arbitrario cultural" y el carácter histórico de su condición. Lo anterior, es calificado por Sayad como una dimensión "epistemológica" de la experiencia migratoria en sus protagonistas, toda vez que se trata de:

(...) la 'relativización' que el emigrado (...) ha experimentado (...) equivale al descubrimiento no sólo del "arbitrario" cultural, (...), sino también de la historicidad de esta condición, es decir, a asignarle un origen y una génesis social y, por consiguiente, una significación histórica. (Sayad, 2010, p. 139)

Una lectura que hacemos de la formulación de Sayad (2010) sobre el "descubrimiento" de la historicidad de la condición de migrante, es como experiencia de descentramiento del yo, la cual se asocia a un sentimiento de extrañeza. Es decir, que no toda la respuesta a la pregunta identitaria ¿Quién soy? en el trayecto migratorio, deviene de las certezas imaginarias de auto-determinación, sino de una historia de emigración e inmigración que lo antecede y atraviesa. Ahora bien, lo anterior se asocia a un sentimiento de extrañeza que está vinculado no sólo a la alteridad social y cultural con que se enfrenta en su trayecto, sino a un extrañamiento respecto a sí mismo en este trayecto de encuentro a la alteridad; que puede dislocar o no, sus referentes de identificación y localización subjetiva. Lo anterior, puede ciertamente tomar una deriva angustiosa, y en algunos casos, entramado a hitos biográficos, podría configurarse como un cuadro psicopatológico.

Ahora bien, los referentes de identificación los concebimos, siguiendo a Freud (1979), como una instancia de articulación entre cultura y psiquis. Así, el ideal del yo se forma con los ideales culturales y sociales incorporados, asumidos y experimentados como propios por el yo. Lo anterior se expresa, como lo explica Bleichman (2010, p. 13), en “(...) coagulaciones discursivas de lo que 'soy', lo que 'no soy', lo que 'debo ser' y lo que 'no debo ser', (...), del modo con el cual la cultura de pertenencia regula las intersecciones entre deseos y sus modos de producción de subjetividad". Es decir, se trata de la pertenencia a un nosotros nacional, etno-racial, de género, de clase, etc., normativo y regulador, según la adscripción a los ideales culturales. De tal modo que "(...) si la representación de sí misma se aproxima al Ideal, la persona experimenta una satisfacción narcisista; si aumenta la distancia entre el Yo y el 
Ideal, tanto mayor será el sufrimiento narcisista" (Tubert, 2010, p. 162). Estos ideales culturales, sin embargo, no se organizan de manera neutra sino que están atravesados por jerarquías de poder, de manera tal que se constituyen como categorías de desigualdad (nación, género, clase y etno-raciales) que se interseccionan (Collins, 2000).

Así, ubicado en un orden de jerarquías según el discurso sobre la migración en la sociedad de llegada, el migrante de tal o cual país, color de piel, etc., tendrá un lugar social con el cual se le identifica. Lo anterior toma la forma del malestar cuando, como lo describe Sayad (2010, p. 364) se trata:

(...) del malestar que conoce quien se siente traicionado por su cuerpo (y también por su nacionalidad) así como todo lo que en él está sometido a representación,(...), el nombre, el lenguaje, el acento y, más ampliamente, todo lo que se llama "cultura", esa marca a la vez escondida y manifiesta (...).

Es decir, aquellas marcas de identificación que en la sociedad de llegada se sobredeterminan y se los confina a un cierto lugar social.

Sin embargo, no todo es del orden de la pérdida y el déficit en la condición del sujeto inmigrante. Siguiendo la línea de Mezzadra (2005) sobre los excedentes (los elementos de autonomía) de los movimientos migratorios contemporáneos, se pueden dar procesos en los cuales los sujetos producen pertenencias problemáticas, tanto para sí mismos como para las culturas y sociedades en las que se inscriben. Es decir que impugnan, con sus pertenencias dobles o triples, el imperio de lo Uno (Laplantine, Nouss, 2007), es decir, de pertenecer a un sólo lugar. A la vez, pueden producirse pertenencias paradojales (Venturini, 2006), en las que el sujeto se incluye y excluye a la vez como extranjero, sin que aquello les resulte un déficit. Muy por el contrario, de aquello sacan ventajas en tanto, les permite moverse con facilidad entre contextos y códigos sociales y culturales distintos.

Entonces, si bien en el trayecto migratorio se dan procesos en los cuales emerge la angustia y se configuran síntomas, éstos no constituye necesariamente y en cualquier condición de migración, un cuadro psicopatológico. Es así como el propio Atchotegui (2012) reconsideró, a partir de las críticas recibidas, el campo de aplicación de su categoría diagnóstica para los migrantes, limitándola a quienes migraban en situaciones de precariedad y violencia. De tal manera, el foco recae en los efectos de la violencia en los sujetos y ya no en la experiencia migratoria en sí.

La migración por refugio es una situación particular de movilidad, atravesada en alguna medida por situaciones de violencia política y social. Muchas veces, las personas se ven forzadas a salir de sus países por amenazas que ponen en riesgo su vida, su integridad física y/o la de sus familias. En estas situaciones, tienen que salir lo más rápido posible y no alcanzan a planificar 
ni proyectar las condiciones en las que vivirán en el nuevo contexto. Estas condiciones particulares de la migración por refugio plantean desafíos en la atención clínica que hemos esbozado en un artículo anterior (Lara et alii, 2017) y que aquí mostraremos a la luz de un caso clínico.

\section{Atención a personas en situación de Refugio}

Tal como se señala en el sitio web institucional de ACNUR-Chile ${ }^{2}$, este país es un Estado Parte de la Convención de 1951 sobre el Estatuto de los Refugiados y de su Protocolo Adicional de 1967. Sin embargo, no fue sino hasta el año 2010 que fue aprobada la "Ley 20.430 de Protección al Refugiado"$^{\prime 3}$ en el país, la cual establece una normativa integral para regular la protección de las personas refugiadas.

Como hemos dicho, en la migración por refugio se trata de aquellos casos en los cuales las personas se ven forzadas a migrar por situaciones de violencia en los países de proveniencia. Así en la Ley de Refugio, tomando la definición se la Declaración de Cartagena (1984), se establece que podrán solicitar refugio en Chile quienes:

(...) hayan huido de su país de nacionalidad o residencia habitual y cuya vida, seguridad o libertad han sido amenazadas por la violencia generalizada, la agresión extranjera, los conflictos internos, la violación masiva de los derechos humanos u otras circunstancias que hayan perturbado gravemente el orden público en dicho país. (Ley 20.430)

El proceso para establecer la condición de Refugio en Chile, está a cargo del Departamento de Extranjería y Migración, quien recibe las solicitudes de refugio y analiza los casos, los que son presentados ante la Comisión de Reconocimiento. En esta instancia, se inicia un proceso en el cual el solicitante de refugio debe dar una serie de entrevistas, para demostrar la veracidad de su relato y la legitimidad de sus solicitud.

En paralelo a este proceso de reconocimiento, la agencia implementadora de ACNUR en Chile, la Fundación de Ayuda Social de las Iglesias Cristianas (FASIC), presta asesoramiento, asistencia humanitaria básica y apoyo, para facilitar la autosuficiencia de las personas refugiadas y solicitantes de refugio en todo Chile.

Tal como consigna ACNUR Chile, el país ha recibido a cerca de 7000 personas refugiadas y solicitantes de refugio, provenientes de diferentes países

ACNUR Chile. Página web institucional. Disponible en: <https://www.acnur.org/chile.html>. Consulta en: 14.12.2018.

3 Ley 20.430 De Protección al Refugiado, Publicada en el Diario Oficial el día 15 de Abril de 2010. Disponible en: <https://www.acnur.org/fileadmin/Documentos/BDL/2010/7733.pdf>. Consulta en: 17.12.2018. 
de América Latina, África, Asia, Europa, donde la mayoría son de nacionalidad colombiana. A la vez, desde el año 1999, Chile inició un Programa de Reasentamiento en el cual han participado centenares de refugiados de origen afgano, palestino, yugoslavo, sirio y colombiano. Este Programa está a cargo de la Vicaría de la Pastoral Social de Caritas.

CEAC ha estado en convenio desde el año 2010 con diversas agencias implementadoras de ACNUR, quienes derivan a atención psicológica a las personas que, mediante una entrevista, determinan que lo requieren. De tal modo, se trata de pacientes que llegan a consultar a través de una derivación institucional, ya que rara vez consultan de manera espontánea. Esto hace que, en un primer momento, sea necesario establecer con cada paciente sus propios motivos de consulta, los cuales pueden coincidir o no con los de la institución derivante.

Como hemos dicho, diversos nudos críticos emergen en la atención clínica a personas en situación de refugio. Por una parte, el haber estado expuesto a situaciones de peligro o de amenaza inminente de su vida hace que en Chile, en un primer momento, se encuentren en un estado de constante expectación y miedo, no sólo respecto a las amenazas hacia ellos que podrían repetirse ${ }^{4}$, sino hacia sus familias que han quedado atrás.

En muchos casos, el haber viajado solos a Chile dejando a sus familiares, genera sentimientos de culpa respecto a las lealtades familiares, que les dificulta poder establecerse subjetivamente en Chile. Un paciente, Juan ${ }^{5}$ de Colombia, refería tener sentimientos de culpa por haber sido él quien saliera de su país, salvando su vida, pero dejando a los miembros de su familia en peligro. Lo anterior, generaba angustia y miedo cotidiano a recibir noticias fatales cuando tenía contactos telefónicos con ellos, evitando así dichos contactos y aumentando las fantasías de culpa y castigo.

Otro elemento crítico, es la situación de precariedad social y económica en que se encuentran muchos de quienes solicitan la condición de refugio al estado chileno. Esta solicitud, según los gobiernos de turno, puede llegar a demorar uno o dos años en resolverse. Durante este tiempo de espera, se encuentran con un número de identificación nacional provisorio, lo cual genera sospechas en los posibles empleadores. Con aquello, se reducen considerablemente las posibilidades de ser contratado en trabajos formales, por lo que sólo les queda tomar trabajos informales con bajos sueldos y sometidos, en la mayoría de los casos, a horarios de trabajo extenuantes. Sin

\footnotetext{
4 En esta oportunidad no nos referiremos a los casos que presentan sintomatología asociada al cuadro de estrés post-traumático.

5 Los datos de identificación han sido modificados para resguardar la confidencialidad del paciente.
} 
embargo, para muchos resulta un alivio estar ocupados la mayor parte del día. Así es como Juan señalaba que, tener sus pensamientos ocupados en el trabajo, le distraía de las preocupaciones respecto a la familia que quedó en su país. A la vez que, llegar extenuado después de largas jornadas laborales y durante los fines de semana, le era funcional a sus sentimientos de culpa, en la medida en que era significado por él como un castigo que le aliviaba.

Una situación distinta es cuando los síntomas de angustia y culpa les impiden concentrarse en sus labores cotidianas en el trabajo y por tanto, tienen la sensación de no poder hacer. Así, cuando aquel malestar constituye una limitación para trabajar, la demanda de atención se manifiesta de manera espontánea. Cuando esto ocurre, muchas veces, han pasado meses en que intentaron sobrellevar la angustia, ahondando el estado de aislamiento, desánimo y sensación de invalidez respecto a su propósito migratorio.

Ahora bien, en dicho aislamiento y falta de redes de apoyo, muchas veces incide la desconfianza que se tiene de los connacionales presentes en Chile, ya que se les considera que podrían estar vinculados a las redes de extorsión que los llevó a dejar su país. Por tanto, se considera un riesgo tomar contacto con la comunidad de las personas de su país en Chile. Independientemente de que aquello pudiera ser un riesgo real o no, es decir, que efectivamente el alcance de las redes de extorsión llegara a Chile, lo que se identifica es la desconfianza básica en el otro, de sus intenciones, y por tanto donde vincularse a alguien desconocido resulta un riesgo. Lo anterior, constituye un impedimento para la instalación de la transferencia de trabajo clínico, en la medida en que ésta arranca del supuesto de que el otro, su conocimiento clínico a través de la palabra, puede curar al paciente de sus padecimientos (Laplanche y Pontalis, 2004). Por tanto, en los casos de solicitantes de asilo y refugiados que han estado expuestos de manera repetida y sistemática a situaciones de violencia, se ha quebrado aquello que simbólicamente permite suponer que tanto el otro semejante, como el Otro del Estado y sus instituciones, no nos destruirá con su hostilidad ("Pacto social" de Hobbes). De este modo, aparecen en ellos dificultades para vincularse $y$, sobre todo, la desconfianza en la palabra, en aquello que nos constituye y permite la relación con otros.

En el caso de María ${ }^{6}$, que expondremos a continuación, fue especialmente evidente la cuestión de la desconfianza en el otro y la palabra, para la posibilidad de su instalación transferencial.

María fue derivada a psicoterapia un mes después de llegar desde Colombia, por una de las instituciones de iglesia encargada en esos años, de proveer apoyo psicosocial y económico a las personas en condición de refugio

${ }_{6}$ Los datos de identificación han sido modificados para resguardar la confidencialidad de la paciente. 
en Chile. La trabajadora social que la había entrevistado explicitaba como motivo de derivación el aislamiento, miedo y desánimo que presentaba, así como irritabilidad y rabia por estar en Chile. En la primera entrevista, María se presentó como una mujer de clase acomodada en la sociedad bogotana, casada y con dos hijos.

Ella hablaba de su marido con mucha irritación y sentimientos de fastidio. En el relato de las discusiones que tenía con él, ella se ubicaba en una posición de superioridad respecto a él. A la vez que se describía como habiendo sido criada por su padre como una "niña mimada" en su juventud. Así, recordaba que en esos años, sentía "que nada malo me pudiera suceder, porque era hija de mi padre". Aquello, tomaba la forma "estar blindada" de los ataques de otros por ser hija de su padre, un hombre respetado y temido. Además, aquello le permitía incurrir en excesos de diverso tipo sin consecuencias, ya que él la defendería ante los regaños de su madre.

A poco andar, a esta imagen de omnipotencia vinculada a su padre, se contrapone una de niña huérfana, al señalar que a su padre biológico casi no lo conoció. A quien ella nombraba como su padre, era un familiar consanguíneo que la había adoptado como su hija. Su madre había tenido una relación casual con el padre biológico de ella y, al poco tiempo de nacida, él falleció. Desde ahí, la madre había trabajado mucho para asegurar que "no me faltara nada".

De esta manera, la paciente iba desplegando elementos de su biografía que escogía para presentarse y eludía hablar del motivo de su viaje a Chile por refugio. De manera tal que no se insistió en indagar por aquello en ese momento, en consideración a que había pasado recientemente por la entrevista de Reconocimiento, en la cual había sido interrogada por las situaciones que motivaban su solicitud. Situación que, como señaló en sesión, aumentaba su angustia y sensación de vulnerabilidad.

Es así como al cabo de unos tres meses, al entrar a sesión visiblemente angustiada, expresa que necesita preguntar algo. Y señala: "tú vas a creer que estoy loca, pero necesito preguntártelo, ihay alguna cámara o grabadora en esta oficina?" sin disimular la sorpresa ante su pregunta la respuesta fue "No". A lo cual ella manifestó su alivio y vergüenza al mismo tiempo, explicando: "En mi país había una institución del Estado de la que se supo hace poco, en el siguiente gobierno, que estaba involucrada con paramilitares, entonces pensé que tu podías estar transmitiendo lo que yo digo aquí, al consulado de mi país". A partir de dicha revelación de su desconfianza, María pudo comenzar a relatar las situaciones vividas por ella y su marido que los hicieron solicitar refugio en Chile, por "ser el país de América latina más lejano de Colombia, junto con Argentina, pero más desconocido para los colombianos". 
A partir de ese momento, María fue relatando las experiencias de violencia vividas a partir de la muerte de su padre, y la manera en que se fue acostumbrado a aquello. De modo tal que ante el descenso de su status económico en Chile, se revelaba con rabia, culpando a su marido de haberla convencido de viajar. Ella verbalizaba que a veces, habría preferido quedarse allá. Sin embargo, en otras ocasiones, se desdecía de aquello con sensación de vergüenza y reflexionaba en torno a la paradoja de que su padre asesinado, habiendo sido quien cumplía la función de protegerla, de algún modo que ella no terminaba de comprender, la había puesto en una situación en la que estaba en peligro su vida.

En el proceso de María, el haber podido verbalizar la desconfianza en las instituciones que se suponía la estaba apoyando en Chile, como una posible reedición de la situación en que la dejó su padre, fue un punto de inflexión en su instalación transferencial. A partir de allí, se fue desplegando un proceso de tres años en el cual, su constancia le permitió restituir el lazo social en Chile.

Así es como en muchos casos de refugio y otros marcados por experiencias de violencia, la instalación de una trasferencia de trabajo clínico puede tomar más tiempo de lo esperado. Por tanto, resulta relevante no dar por sentado que ésta se ha establecido antes de tiempo.

Durante el período que siguió, María oscilaba en su relato entre estar agradecida de su marido por haberla traído a Chile, donde estaba más tranquila, y verbalizar sus sentimientos de rabia hacia él, porque ella no quería dejar Colombia. Durante varias sesiones ella describió su vida en Bogotá, en la cual no había límites en la tarjeta de crédito, sólo trabajaba esporádicamente en un negocio familiar y se dedicaba a cuidar y vestir con prolijidad a su hija mayor. Al Ilegar a Chile sin embargo, las condiciones fueron muy distintas. A pesar de estar lejos de la precariedad en que se encontraban otros inmigrantes y refugiados de su país, cuando iba a la institución implementadora de los apoyos de ACNUR en Chile, salía con mucho enojo por estar incluida en el grupo de "refugiados, pobrecitos a los que hay que ayudar".

De modo tal, María recorrió un proceso de reconfiguración donde sus referentes de identificación de clase, ya no la ubicaban en la clase media-alta de Bogotá sino, media o media-baja, como ella misma se identificaba en Santiago. Para relocalizarse en su adscripción de clase, ella tomaba como referencias, el lugar en la ciudad donde vivía, el trabajo de su marido, el colegio de sus hijos, etc. Dichas referencias las manejaba muy bien, ya que en su biografía había tenido una trayectoria de ascenso social en Bogotá, vinculado a no-tener/tener un padre. De modo que, en el primer tiempo de su inmigración a Chile, no sólo se quejaba del descenso socieconómico que estaba experimentando, sino que se resistía a aquello, prefiriendo incluso no haberse ido de Colombia y haber seguido viviendo en la violencia cotidiana. 
En un inicio, ella no se resignaba a perder aquello que sostenía su posición de omnipotencia narcisista, pero una vez asumido que ya no era la "niña mimada", apareció la tristeza del duelo no elaborado. Fue relevante trabajar aquello para que, a partir del evento del refugio, ella resinificara aquellos hitos biográficos y los conflictos en que estaba situada.

Otro registro en el que se jugaba la desconfianza, así como la reconfiguración identitaria de clase y nacionalidad en este caso, fue en el de las palabras que utilizaba durante la sesión. Por una parte, en el primer tiempo, María preguntaba constantemente si la estaba entendiendo al hablar. De manera que, a pesar de puntualizar que si no entendía alguna de sus palabras se lo preguntaría, había una desconfianza constante a que la pudiera entender en su manera de hablar.

María apuntaba que habían escogido venir a Chile, además de por la estabilidad política y económica del país, porque a diferencia de Brasil, se hablaba español. Sin embargo, se había dado cuenta que también había diferencias entre el idioma español de Colombia y Chile. Así, la desconfianza se materializó en el uso de las palabras y las entonaciones al hablar: "Me impactó mucho que al principio, cuando le preguntaba a una persona de la calle ino le entendía nada!, es que hablan muy rápido y usan una entonación que me confunde. No sabía si lo que decían era una pregunta o una afirmación". En el caso de María, aquello suscitaba una angustia inesperada para ella, en la medida en que no se trataba solamente de la inseguridad para entender las palabras y entonaciones del otro chileno, sino una desconfianza en las intenciones del otro al hablar, es decir, de no querer ayudarla ni darse a entender. A la vez, como hemos dicho, no confiaba en personas de su misma nacionalidad, como mujeres colombianas que conocía en la plaza estando con sus hijos. De este modo, en su primer tiempo en Santiago, María sólo salía del departamento en que vivía para ir a dejar y a buscar a sus hijos al colegio y más tarde para ir a sesiones semanalmente.

Muchas de aquellas sesiones del primer tiempo de María en Santiago, se centraban en el relato de los momentos de angustia en su vida cotidiana, al encontrarse con otros de quienes desconfiaba. En aquellas situaciones, se trataba de interacciones que tenían la posibilidad el engaño, el abuso o la violencia: el taxista que la podría engañar o incluso raptar, la manifestación estudiantil donde podría ser confundida con una manifestante y golpeada por la policía, la mujer colombiana en la plaza que podría estar vinculada a las redes de extorsión. En todas aquellas situaciones, ella sentía una angustia que la invalidaba para defenderse o reaccionar, con lo cual evitaba salir y se replegaba al pequeño departamento donde se sentía un poco más segura y desanimada.

En ese primer período de su vida en Santiago, María explicitaba su confusión e inseguridad con las palabras. Así, relataba momentos en que 
se daba cuenta que las palabras, siendo las mismas que se usan en Chile y Colombia, tienen significados distintos. Lo anterior, también sucedía en las sesiones, donde ella usaba la palabra "pena", con el significado de "vergüenza", y era escuchada como "tristeza", lo cual podría llevar a confusiones y aumentar su sensación de desconfianza en que pudiera entenderla. La manera de trabajar aquello, fue no sobreentender sus palabras y explicitar la ambigüedad, expresando que al parecer, estábamos entendiendo significados distintos con una misma palabra. En estos diálogos sobre sus palabras, sus modismos y maneras de decir, así como los que yo misma entendía y usaba, íbamos tejiendo un lazo en el cual no se trataba de un "problema de comunicación", sino de su ubicación subjetiva en estas palabras y sus diferencias. De restituir la confianza en la palabra y el otro, en un encuentro contingente de alteridad. Así como los elementos de su vida cotidiana en Santiago, que la reenviaban a su historia, resinificada desde ese presente.

\section{Conclusión}

La perspectiva de trabajo clínico de AMIR se funda en la premisa de que los malestares, síntomas y sufrimientos de los migrantes no se reducen sólo a una etiología cultural, sino al entramado de dimensiones socioculturales, políticas y psíquicas que componen una subjetividad. Por tanto, nos alejamos de aquellas perspectivas de trabajo clínico con migrantes que suponen que la experiencia migratoria es traumática en sí misma, por la salida del sujeto de su cultura, sino que su potencial psicopatológico dependerá de las condiciones en que ésta se realice.

De manera tal, planteamos que el trabajo clínico debiera orientarse a abordar los conflictos que, en el proceso de relocalización subjetiva de los migrantes, van emergiendo con dinámicas e intensidades singulares en cada caso, según los entramados biográficos e históricos que los cruzan. Muchas veces, estos conflictos se han reactivado o elicitado en el trayecto migratorio, pudiendo ser reflexionados y elaborados por el paciente con posterioridad a la salida de su país.

Ahora bien, hemos analizado la situación particular de migración por refugio, es decir, donde el motivo está cruzado, las más de las veces, por experiencias de violencia sistemática y sostenida en el tiempo. Por tanto, es fundamental estar advertidos de que la instalación de la transferencia muchas veces pasa, por la reconstitución de la confianza en la palabra, en el otro y en el lazo social, ya que sólo desde allí podrán desplegarse sus conflictividades. Y se los podrá acompañar en los derroteros de su trayecto, para permitir abrir espacio subjetivo a aquello que es del orden de la creatividad y de lo nuevo, es decir, para salir de la repetición de lo mismo. 


\section{Referencias}

ATCHOTEGUI, Joseba. Migración y salud mental, El síndrome del inmigrante con estrés crónico y múltiple (síndrome de Ulises). Revista Zerbitzuan, v. 46, n. 3, p. 163-171, 2009.

ATCHOTEGUI, Joseba. Emigrar hoy en situaciones extremas. El síndrome de Ulises. Aloma, v. 2, n. 30, p. 79-86, 2012.

BECERRA, Margarita; ALTIMIR, Laura. Características y necesidades de las personas migrantes que consultan en salud mental: la emergencia del fenómeno de encuentro intersubjetivo de negociación intercultural. De familias y Terapia, n. 35, p. 101-118, 2013.

BLEICHMAN, Silvia. Desmantelamiento de la subjetividad. Estallido del yo. Buenos Aires: Topía, 2010.

BRIGIDI, Serena. Políticas públicas de Salud Mental y Migración Latina en Barceona y Génova (Tesis de Doctorado). Tarragona, España: Universitat Rovira I Virgili, 2009.

CABIESES Báltica; BERNALES Margarita; MCINTYRE Ana María. Introducción y conceptos esenciales. In: CABIESES, Báltica; BERNALES, Margarita; MCINTYRE, Ana (eds.). La migración internacional como determinante social de la salud en Chile: evidencia y propuestas para políticas públicas. 2017. Disponible en: <http://www.udd.cl/dircom/pdfs/Libro_La_migracion_internacional.pdf > .

COLLINS, Patricia Hill. Gender, Black Feminism, and Black Political Economy. Annals of the American Academy of Political and Social Science, v. 568, n. 1, p. 41-53, 2000.

FALICOV, Celia. Latino Families in Therapy. New York: Guilford Press, 2015.

FREUD, Sigmunt. Psicología de las masas y análisis del yo. Obras Completas, v. XVIII. Madrid: Amorrortu, 1979 [1921].

FREUD, Sigmund. Sobre la dinámica de la trasferencia. Obras Completas, v. XII. Madrid: Amorrortu, 1979 [1912].

FREUD, Sigmund. Fragmento de análisis de un caso de histeria. Obras Completas, v. VII. Madrid: Amorrortu, 1979 [1901].

FREUD, Sigmund. Psicoterapia de la histeria. Obras Completas, v. II. Madrid: Amorrortu, 1979 [1895].

GRINBERG, Rebeca; GRINBERG, Leon. Migración y Exilio. Estudio Psicoanalítico. Madrid: Biblioteca Nueva, 1996.

INE. Censo 2002. Síntesis de resultados. 2003. Disponible en: <https://www. ine.cl/docs/default-source/FAQ/s\%C3\%ADntesis-de-resultados-censo-2002. pdf?sfvrsn $=2>$. Consultado en: 10.12.2018.

INE. Censo de población 2017. Características de la inmigración internacional en Chile. 2018. Disponible en: <http://www.ine.cl/docs/defaultsource/demogr\%C3\%A1 ficas-y-vitales/inmigraci\%C3\%B3n/documentoinmigraci\%C3\%B3n.pdf?sfvrsn=4>. Consultado en: 10.12.2018. 
LAPLANCHE, Jean; PONTALIS, Jean-Bertrand. Diccionario de psicoanálisis. Buenos Aires: Paidós, 2004.

LAPLANTINE, Francoise; NOUSS, Alexis. Mestizajes. De Arcimboldo a Zombi. Buenos Aires: FCE, 2007.

LARA, Antonia; JIMENEZ, Francisco; TOCORNAL, Constanza; CAYUPAN, Eva; GALLARDO, Ivania; MATELUNA, Damaris; ALCAINO, Michelle. Nudos críticos en la atención psicoterapéutica a migrantes y refugiados, desde un centro universitario. Revista Sul Americana de Psicologia, v. 5, n. 2, p. 161-171, 2017.

MARTíNEZ, Jorge. El encanto de los datos: sociodemografía de la inmigración en Chile según censo de 2002. United Nations Publications, 2003.

MEZZADRA, Sandro. Derecho de Fuga. Migraciones, ciudadanía y globalización. Madrid: Traficante de sueños, 2005.

NATHAN, Tobie. La influencia que cura. Buenos Aires: Fondo de Cultura Económica, 1999.

SAYAD, Abdelmalek. La Doble ausencia. De Las ilusiones de emigrado a los padecimientos del inmigrado. Barcelona: Anthropos, 2010.

STEFONI, Carolina. Perfil migratorio de Chile. Santiago: OIM, 2011.

TUBERT, Silvia. Los ideales culturales de la feminidad y sus efectos sobre el cuerpo de las mujeres. Quaderns de Psicología, v. 12, n. 2, p. 161-174, 2010.

VENTURINI, Soledad. La emergencia del sujeto en la migración. Aesthethika, v. 2, n. 2, p. 62-68, 2006. 